\title{
Predominant peak detection of QRS complexes
}

\author{
Ahmed Saad Al-Ghabban, Abbas Fathil Al-Hashimi, Ziad Tariq Al-Dahan \\ Department of Medical Engineering, Alnahrain University, Baghdad, Iraq
}

Email address:

ahmed_alghabban@yahoo.com (A. S. Al-Ghabban)

To cite this article:

Ahmed Saad Al-Ghabban, Abbas Fathil Al-Hashimi, Ziad Tariq Al-Dahan. Predominant Peak Detection of QRS Complexes. International Journal of Medical Imaging. Vol. 2, No. 6, 2014, pp. 133-137. doi: 10.11648/j.ijmi.20140206.12

\begin{abstract}
This work presents a methodology for detection of QRS complexes which is an adaptation of Pan-Tompkins algorithm. This method includes band bass filtration, derivative, squaring, moving window integration, thresholding, false peak cancellation and detection the predominant peak of QRS complex. This method doing well in the presence of noise and simple ectopic beats but still affected by multifocal ectopic beats. The method was tested on the MIT-BIH Arrhythmia database and achieves accuracy $99.68 \%$.
\end{abstract}

Keywords: ECG, QRS, Ectopic beat, Butterworth Filter

\section{Introduction}

The Electrocardiograph (ECG) is the graphical representation of the electrical voltages generated during the cardiac activity. Since it reflects the rhythmic electrical depolarization and repolarization of the atria and ventricles, its shape, time interval and amplitude provide much information about the current state of the heart. One cardiac cycle of ECG consists of the P wave, QRS complex, T wave, small $U$ wave. The diagnosis of cardiac abnormalities is performed based on features extracted from the timing and morphology of these fiducial points. [1]

A good performance of an automatic ECG analyzing system highly depends upon the accurate and reliable detection of the QRS complex. The QRS complex is the most crucial step in automatic electrocardiogram analysis such as arrhythmia detection and classification of ECG diagnosis and heart rate variability studies [2].

One of the dependable method of QRS detection is the Pan-Tompkins method [3]. This method employs band pass filtering and nonlinear processing for ECG pre-processing and thresholding. In this work, detection has been carried out using an adaptation of the Pan-Tompkins algorithm. This adaptation includes using different bass band frequency to eliminate the noise while maintaining the QRS energy as well as different thresholding and detection steps.

\section{Methods}

\subsection{Baseline Wander Cancellation}

The baseline wander is an external, low frequency activity [4] in the ECG which leads to deviation from the isoelectric line as shown in figure (1).

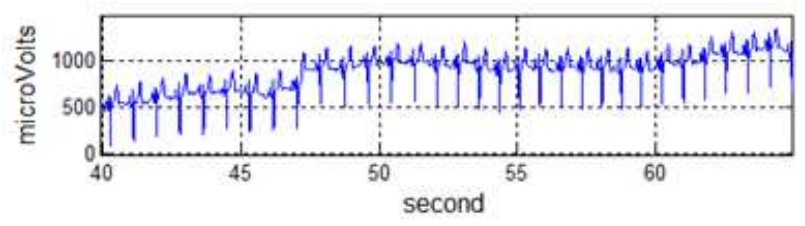

Figure (1). ECG baseline wander

Cancellation of this deviation without affected other ECG waves is important to recognize the predominant peak of QRS. This has been done by FIR high pass filter using Kaiser Window with cutoff frequency of $0.8 \mathrm{~Hz}$ [5]. Figure (2) shows the frequency response of this filter. The output of ECG after baseline wander cancellation denoted by X11

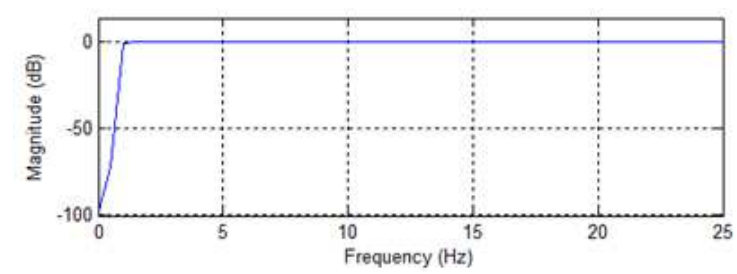

Figure (2). Frequency response of FIR Kaiser Window $H P F$ with $f_{c}=0.8 \mathrm{~Hz}$. 


\subsection{Band Bass Filtration}

QRS peak detection is difficult, not only because of the physiological variability of the QRS complexes, but also because of the various types of noise that can be presented in the ECG signal as well as $\mathrm{P}$ and $\mathrm{T}$ waves with high frequency characteristics similar to QRS complexes.

This filter isolates the predominant QRS energy, attenuates the low frequencies characteristic of $\mathrm{P}$ and $\mathrm{T}$ waves and baseline drift, and also attenuates the high frequencies noise. Experimentally, a range of frequency $(8-25) \mathrm{Hz}$ has been chosen to be the desirable pass band to maintain the QRS energy as maximum as possible as well as to minimize noise and the effects of other ECG waves.

To eliminate frequencies above (25) Hz, a 4th order Butterworth low pass filter with cutoff frequency (25) $\mathrm{Hz}$ has been used. Figure (3), shows the frequency response of this filter. The output of this step denoted by X21



Figure (3). Frequency response of 4th order Butterworth $L P F, f_{c}=25 \mathrm{~Hz}$

To eliminate frequencies less than $8 \mathrm{~Hz}$, FIR high pass filter using Kaiser Window with $\mathrm{fc}=8 \mathrm{~Hz}$ has been used. The figure (4) shows the frequency response of this filter. The output of this step denoted by X22.

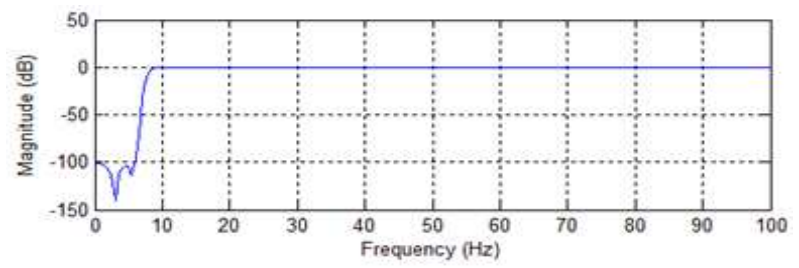

Figure (4). Frequency response FIR Kaiser window $H P F, f_{c}=8 \mathrm{~Hz}$

\subsection{Derivative}

This step includes differentiation which is the standard technique for finding the high slopes that normally distinguish the QRS complexes from other ECG waves.



Figure (5). Frequency response of the derivative filter

The transfer function of five-point derivative filter describe in equation (1), and figure (5) shows the frequency response of this filter.

$$
\mathrm{H}(\mathrm{z})=\frac{2+\mathrm{z}^{-1}-\mathrm{z}^{-3}-2 \mathrm{z}^{-4}}{8}
$$

\subsection{Squaring}

Squaring is a nonlinear transformation that consists of a point-by-point squaring of the signal samples of (X23) and as described in equation (2).

$$
\mathrm{X} 24=[\mathrm{X} 23]^{2}
$$

This transformation serves to make all the data positive prior to subsequent integration, and also accentuates the higher frequencies in the signal obtained from the differentiation process. These higher frequencies are normally characteristic of the QRS complex.

\subsection{Moving Window Integration}

Moving window integration has been used to obtain waveform feature information in addition to the slope of the $\mathrm{R}$ wave. It has been calculated as described in equation 3 .

$\mathrm{Y}(\mathrm{nT})=(1 / \mathrm{N})[\mathrm{X}(\mathrm{nT}-(\mathrm{N}-1) \mathrm{T})+\mathrm{X}(\mathrm{nT}-(\mathrm{N}-2) \mathrm{T})+\ldots .+\mathrm{X}(\mathrm{nT})]$

where $\mathrm{N}$ is the number of samples of the integration window.

The width of the window should be approximately the same as the widest possible QRS complex. If the size of the window is too large, the integration waveform will merge the QRS and T complexes together. On the other hand, if the size of the window is too small, a QRS complex could produce several peaks at the output of the stage. Thus, the width of the window should be chosen experimentally. For the data used in this work with sample rate of $360 \mathrm{~Hz}$, the window chosen for this algorithm was approximate 40 samples wide (which approximately corresponds to $111 \mathrm{~ms}$ ). This step has been applied twice in order to improve the output of this stage which becomes the reference for predominant peak of QRS complex detection. The output of these two steps denoted by X25 and X26 respectively.

\subsection{Thresholding}

After the previous steps of filtrations and obtaining slop information, detection steps has been applied on the output of the integrating step (X26). This threshold calculated as the mean value of (X26) as in equation (4).

$$
\text { THRESHOLD }=\text { mean }(\mathrm{X} 26)
$$

This threshold value has been calculated every 10 seconds.

\subsection{False Peak Cancellation}

The aim of filtration and thresholding were to isolate peaks which refers to QRS complex from the others. Moreover, some spikes, noise and some of the $\mathrm{P}$ or $\mathrm{T}$ waves still pass the threshold which may lead to detect them as a QRS as shown in figure(6) . 




Figure (6). False peaks detected after thresholding

To cancel these false peaks, the difference between the positions of each successive peaks of (X27) has been calculated. Firstly, any value below (0.3) of the mean differences indicate that one of these peaks is wrong, which is mostly the peak with the lowest amplitude peak value.

After that, any value below (0.9) of the mean differences which lies between two peaks with position difference less than (1.1) of the mean difference indicate that one of these peaks is wrong, which is mostly the peak with the lowest amplitude peak value .The mean difference of the peaks determined after any peak cancellation and repeating the previous processing with new mean difference value.
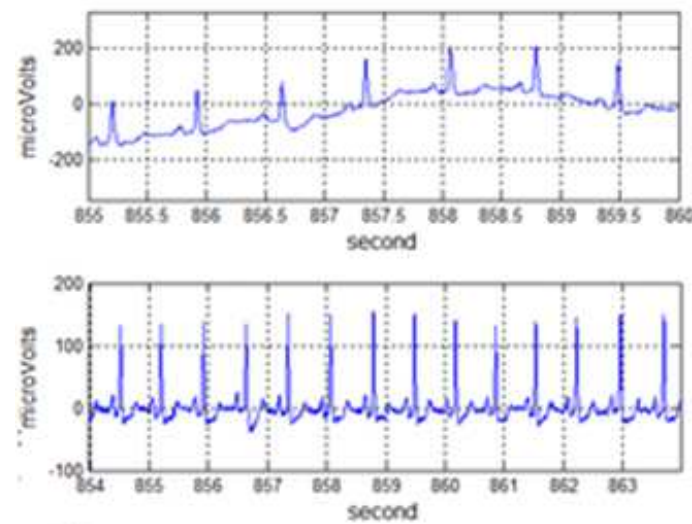

b]


(c)
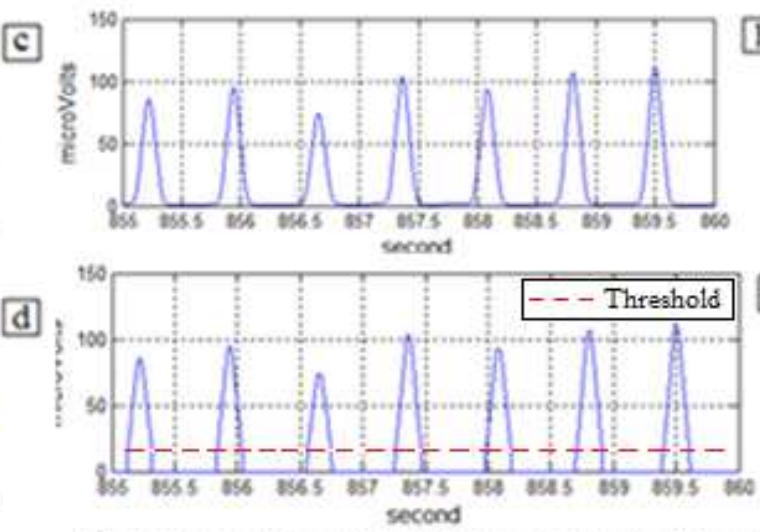

e

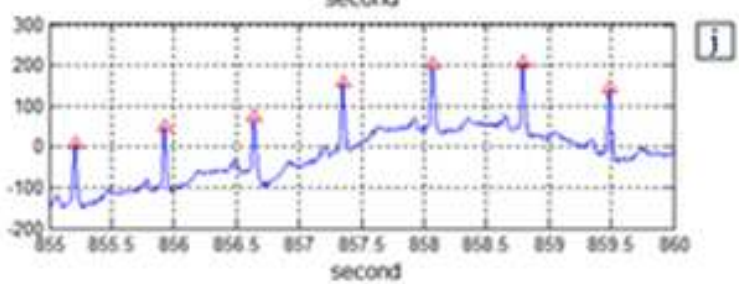

Figure (7). (a). Input ECG signal. (b). Baseline wander cancellationX11. (c) Filtration using Butterworth LPF X21. (d). Filtration using Butterworth HPF X22. (e) Filtration using derivative filter X23. (f) Signal output after using squaring function X24. (g) Signal after moving window integration X25. (h) Signal after moving window integration X26. (i). signal after thresholding. (j) Detection of predominant peak of QRS complex. 
From table 1 it has been found that there are totally 45032 detected heart beats, with 121 missed beats and 21 extra detected points. The averaged accuracy of this method is $99.68 \%$ using the equation (5) [7]:

$$
\text { Accuracy }=1-\frac{F P+F N}{T B}
$$

Where the FP is the number of extra detected points, $\mathrm{FN}$ is the number of missed beats and $\mathrm{TB}$ is the number of total beats.

Table 1. Result of the proposed algorithm applied to MIT-BIH Arrhythmia database.

\begin{tabular}{|c|c|c|c|c|}
\hline Record & TB & FP & FN & Error \% \\
\hline 100 & 2272 & 0 & 0 & 0 \\
\hline 101 & 1867 & 2 & 3 & 0.27 \\
\hline 102 & 2187 & 0 & 0 & 0 \\
\hline 103 & 2083 & 1 & 0 & 0.05 \\
\hline 104 & 2224 & 8 & 4 & 0.54 \\
\hline 105 & 2572 & 10 & 4 & 0.54 \\
\hline 106 & 1944 & 70 & 0 & 2.97 \\
\hline 107 & 2134 & 0 & 0 & 0 \\
\hline 108 & 1757 & 13 & 7 & 1.14 \\
\hline 109 & 2524 & 5 & 0 & 0.198 \\
\hline 111 & 2123 & 0 & 0 & 0 \\
\hline 112 & 2539 & 0 & 0 & 0 \\
\hline 113 & 1794 & 0 & 0 & 0 \\
\hline 114 & 1879 & 2 & 1 & 0.16 \\
\hline 115 & 1952 & 0 & 0 & 0 \\
\hline 116 & 2392 & 1 & 2 & 0.13 \\
\hline 117 & 1535 & 0 & 0 & 0 \\
\hline 118 & 2278 & 0 & 0 & 0 \\
\hline 119 & 1987 & 0 & 0 & 0 \\
\hline 121 & 1862 & 0 & 0 & 0 \\
\hline 123 & 1515 & 2 & 0 & 0.13 \\
\hline 124 & 1612 & 7 & 0 & 0.43 \\
\hline
\end{tabular}

As mentioned before, this method is for the detection of the predominant peak of QRS complexes that is because some times and in some ECG leads R peak not represent the maximum power of QRS complex and not easy to be detected thus, Q or S peak will be considered as a fiducial point as shown in figure 8



Figure (8). Detection of Q peak as fiducial points.
This method has been tested on ECG signal contains an ectopic beats which are an abnormal sequence of contraction of the different parts of the heart leading to contraction of the heart before the time that normal contraction would have been expected [8] as shown in figure 9.

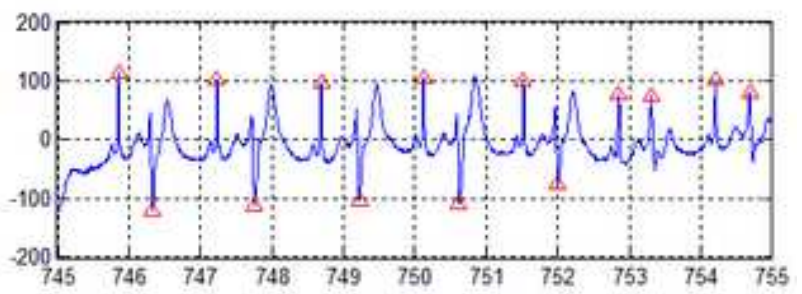

Figure (9). Detection of predominant peak of QRS complex of ECG signal contains ectopic beats

ECG signal with multifocal ectopic beat has also been tested and the result was one of these beats has been missed as shown in figure 10 .

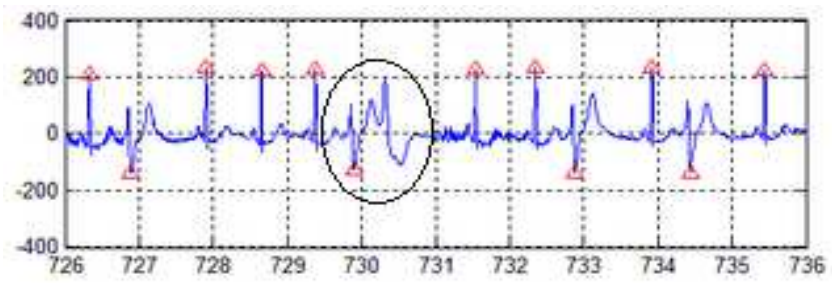

Figure (10). Detection of predominant peak of QRS complex of ECG signal contains multifocal ectopic beat.

\section{Discussion}

The first five steps of this method which were mainly a filtration steps depends on Pan Tompkins algorithm with some adaptations. The main adaptations was the bass band frequencies which was (8-25) Hz instead of (5-11) Hz. It has been found that the range $(5-11) \mathrm{Hz}$ highly weakens the energy of QRS complex which may cause QRS to be at a level asymptotic to $\mathrm{P}$ or $\mathrm{T}$ wave, while the range $(8-25) \mathrm{Hz}$ maintain the QRS energy.

Other adaptation was the using of moving window integration filter for twice. It was found that some noisy and abnormal QRS have a rippled waves after applying the moving window integration filter as shown in figure 11.

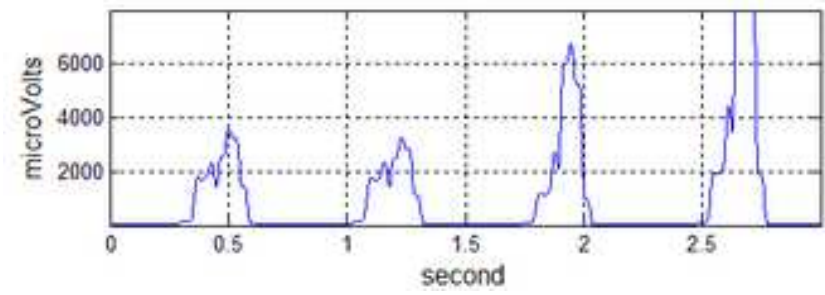

Figure (11). Rippled wave after applying integration filter (X25).

This ripples may lead to great error in case the threshold pass through it thus, the moving integration filter has been applied again to terminate this ripple as shown in figure 12 . 




Figure (12). Signal after integration filter for the second time(X26)

The other steps of this method which were the thresholding and the detection steps are completely different from Pan Tompkins algorithm.

Table 1 shows that this method did perfectly with some records without any missing beats or extra detecting points. Other records contain some missing beats. These beats either had low level amplitude which was less than threshold or some high level noise, spikes or T waves or P waves with an amplitude higher than QRS complex which may lead to keep them and cancel these beats in the false peak cancellation step of this method.

Also it has been found that record (106) had the largest false beats which was greater than have of the total false beats. That was because this record contains many multifocal ectopic beats. The multifocal ectopic beats appear in nonregular time which have been canceled in the false peak cancellation step of this method. Other signal which contain simple ectopic beats has been detected correctly.

As compare the result of our work with the result of the Pan-Tompkins methods mentioned in [3] it has been found that there are totally 45169 detected heart beats with 291 missed beats and 100 extra detected points. The averaged accuracy of this method was $99.13 \%$ while the accuracy of this work is $99.68 \%$.

\section{Conclusion}

After applying this method on different types of data it has been found that it did well with normal ECG even with the presence of noise unless it cause spikes with an amplitude close or greater than the amplitude of QRS complexes .This method also did well with ECG signal that carry simple ectopic beats but ECG with multifocal ectopic beats cause some missing beats.

\section{References}

[1] Chen W. Mo Z. Guo W. "Detection of QRS Complexes Using Wavelet Transforms and Golden Section Search", Biomedical Engineering, Issue 4 , Page 748-751,2009

[2] Sumathi S., Sanavullah M. Y. , "Comparative Study of QRS Complex Detection in ECG Based on Discrete Wavelet Transform" , International Journal of Recent Trends in Engineering, Vol 2, No. 5, November 2009

[3] Pan J., Tompkins W. , "A Real-Time QRS Detection Algorithm" , IEEE TRANSACTIONS ON BIOMEDICAL ENGINEERING, VOL. BME-32, NO. 3, page 230-236,1985

[4] Clifford G.D. , Azuaje F. , McSharry P. E. , Advanced Methods and Tools for ECG Data Analysis, ,ARTECH HOUSE.2006.

[5] Van J.., Van W., Herrmann E. , ECG Baseline Wander Reduction Using Linear Phase Filters, COMPUTERS AND BIOMEDICAL RESEARCH, 19, 1986, pp.417-427, 1986.

[6] Tompkins W.J., "Biomedical Digital Signal Processing" Prentice Hall (Englewood Cliffs, N.J.), 2002.

[7] Yeh Y., Wang W. ,"QRS complexes detection for ECG signal: The Difference Operation Method ", Computer Methods and Programs in Biomedicine, Vol 91, Issue 3 , page 245-254, 2008

[8] Arthur C. Guyton, M.D., John E. Hall, Ph.D. , " TEXTBOOK of Medical Physiology", eleventh edition, Elsevier, 2006. 